\title{
Thermodynamic Properties of Liquid Carbon Tetrafluoride
}

\author{
Léllo Q. Lobo* \\ Departamento de Quimica, Universidade de Coimbra, 3000 Coimbra, Portugal
}

Llonel A. K. Staveley

Inorganic Chemistry Laboratory, University of Oxford, Oxford OX1 $3 Q R$, England

\begin{abstract}
Avallable thermodynamlc data on $\mathrm{CF}_{4}$ have been collected and their reliabllity examined. They have been used to calculate the values of the following properties: (1) the enthalpy of vaporization of the liquid from the triple point $(89.56 \mathrm{~K})$ to $220 \mathrm{~K}$; (2) the configurational internal energy of the liquid and gas at the saturation vapor pressure, and the adlabatic compressibility of the llquid over the same range; (3) the coefficient of expansion of the llquid from the triple polnt to $190 \mathrm{~K} ;$ (4) the ratio $C_{p} / C_{v}$, the heat capaclty at constant volume, the thermal pressure coefficlent, and the Isothermal compresslbility of the Ilquid from $90 \mathrm{~K}$ to the normal bolling point (145.16 K).
\end{abstract}

\section{Introduction}

We have measured in recent years the primary thermodynamic excess properties $-G^{\mathrm{E}}, H^{\mathrm{E}}$, and $V^{\mathrm{E}}$ - of a number of binary mixtures of liquefied gases. The experimental results have been used to test modern theories of liquid mixtures, and in particular the perturbation theory developed by Gubbins and co-workers. For such a theory to be successful it is essential that the intermolecular potential of each one of the pure components be known as accurately as possible. Powles, Gubbins, and collaborators in their study of hydrogen chloride (1) indicated the reasons that a knowledge of the configurational internal energy of the liquid as a function of temperature is useful in improving the intermolecular potential function, and stressed the need for such data. Results of this kind have recently become available for hydrogen chloride from high-pressure $P-V-T$ measurements (2).

As calculations concerning the system $\mathrm{HCl}+\mathrm{CF}_{4}$ are now in progress (3) with the object of comparing experiment and theory for this system, and since we have already measured in this (Oxford) laboratory the vapor pressure (4) and the density (5) of liquid carbon tetrafluoride over wide temperature ranges, it seemed worthwhile to calculate the internal configurational energy of $\mathrm{CF}_{4}$ along the saturation line and the values of some other derived thermodynamic quantities. For this purpose one needs, of course, other information, and we shall briefly comment on the available data and on the methods actually used in the calculations.

\section{Calculations}

The internal configurational energy of a pure liquid $U^{*}$ at temperature $T$ and the saturation pressure $P_{s}$ is the difference between the internal energy of the liquid and that of a perfect gas at the same temperature:

$$
\begin{aligned}
U^{\ddagger}\left(T, P_{\mathrm{s}}\right) & =U_{(}\left(T, P_{\mathrm{s}}\right)-U_{\mathrm{pg}}\left(T, P_{\mathrm{s}}\right) \\
& =U_{(}\left(T, P_{\mathrm{s}}\right)-U_{\mathrm{pg}}(T, 0)
\end{aligned}
$$

where the subscript pg refers to the perfect gas.

When one assumes that the vapor in equilibrium with the liquid follows the virial equation of state

$$
P V_{g} / R T=1+B^{\prime} P+C^{\prime} P^{2}+\ldots
$$

where

$$
B^{\prime}=B / R T \quad C^{\prime}=\left(C-B^{2}\right) /(R T)^{2}
$$

and so forth, and $B, C, \ldots$ are the second, third, ... (volume) virial coefficients, $U^{*}$ is then given by the expression

$$
\begin{aligned}
& U_{1}^{*}\left(T, P_{\mathrm{s}}\right)=-\Delta H_{\mathrm{v}}+R T-V_{1} P_{\mathrm{s}}+ \\
& \quad\left(B-B_{1}\right)\left(P_{\mathrm{s}}-B P_{\mathrm{s}}{ }^{2} / R T\right)+\left(2 C-C_{1}\right)\left[P_{\mathrm{s}}{ }^{2} /(2 R T)\right]
\end{aligned}
$$

neglecting all the virial coefficients after the third. In this equation

$$
B_{1}=T(\mathrm{~d} B / \mathrm{d} T) \quad C_{1}=T(\mathrm{~d} C / \mathrm{d} T)
$$

$\Delta H_{v}$ is the enthalpy of vaporization, and $V_{1}$ is the molar volume of the saturated liquid.

Therefore, to carry out the calculation of $U^{*}$, one should have, at least, values of $V_{1}$ and of the virial coefficients $B$ and $C$ (and their temperature coefficients), and reliable values of $\Delta H_{v}$. One source of these is an appropriate vapor-pressure equation such as the Wagner equation

In $P_{s}=\ln P_{\mathrm{c}}+\left(A_{1} \tau+A_{2} \tau^{1.5}+A_{3} \tau^{3}+A_{4} \tau^{B}\right) / T_{\mathrm{R}}$

where $\tau=\left(1-T_{R}\right)$, and $P_{c}$ and $T_{R}$ are the critical pressure and the reduced temperature, respectively. Ambrose (6) has demonstrated that this equation applies over a wide temperature range, and in fact the widest possible one spanning the triple point to the critical temperature. In the fitting of our experimental values of $P_{\mathrm{s}}$ to eq 6 to obtain the parameters $A_{l}$ (4), the constraints discussed in Ambrose's paper (6) were adopted, so that values of $\Delta H_{v}$ derived from the ClausiusClapeyron equation

$$
\mathrm{d} P_{\mathrm{s}} / \mathrm{d} T=\Delta H_{\mathrm{v}} /\left[T\left(V_{\mathrm{g}}-V_{\mathrm{l}}\right)\right]
$$

should conform closely to experiment over a large temperature interval. An accurate calorimetric value of $\Delta H_{\mathrm{y}}$ at the normal boiling point of $\mathrm{CF}_{4}$ was obtained by Smith and Pace (7), and it is important to note that the value of $\Delta H_{v}$ at the same temperature obtained from eq 7 and the Wagner equation for $\mathrm{CF}_{4}$ agrees virtually exactly with the calorimetric value.

Experimental second virial coefficients $B$ of carbon tetrafluoride given by Lange and Stein (8) (from -70 to $95^{\circ} \mathrm{C}$ ) and by Douslin et al. ( 9 ) (from 0 to $350^{\circ} \mathrm{C}$ ) were fitted by orthogonal polynomials to an expression of the type

$$
B / V_{\mathrm{c}}=\sum_{i} a_{i} T_{\mathrm{R}}{ }^{i}
$$

up to the fourth degree (the standard deviation being $\sigma(B)=$ $\left.\pm 0.1 \mathrm{~cm}^{3} \mathrm{~mol}^{-1}\right)$. Its use on extrapolation to lower temperatures should give reasonably reliable values of $B$, as experience with other substances has shown (10).

As for the third virial coefficient $C$, the equation proposed by Chueh and Prausnitz (11) was used. An estimate of parameter $d$ in this expression was obtained by using experimental values of $C(8,9)$ in a plot of $C / V_{c}^{2}$ as a function of $T_{R}$ and com- 
Table I. Thermodynamic Properties of Liquid and Gaseous Carbon Tetrafluoride ${ }^{a}$

\begin{tabular}{|c|c|c|c|c|c|c|c|c|c|}
\hline$T, \mathrm{~K}$ & $P_{\mathrm{s}}, \mathrm{kPa}$ & $\begin{array}{c}-B, \mathrm{~cm}^{3} \\
\mathrm{~mol}^{-1}\end{array}$ & $\begin{array}{l}C, \mathrm{~cm}^{6} \\
\mathrm{~mol}^{-2}\end{array}$ & $\begin{array}{c}V_{\mathrm{l}}, \mathrm{cm}^{3} \\
\mathrm{~mol}^{-1}\end{array}$ & $V_{\mathrm{g}, \mathrm{dm}^{3}}$ & $\begin{array}{c}\Delta H_{\mathrm{v}}, \mathrm{kJ} \\
\mathrm{mol}^{-1}\end{array}$ & $\begin{array}{c}-U^{\ddagger}{ }_{1}, \mathrm{~kJ} \\
\mathrm{~mol}^{-1}\end{array}$ & $\begin{array}{c}-U^{\ddagger}, \mathrm{kJ} \\
\mathrm{mol}^{-1}\end{array}$ & obsd \\
\hline 89.56 & 0.109 & 718 & & 46.727 & 6830.7 & 14.260 & 13.515 & 0.000 & triple point \\
\hline 90 & 0.120 & 714 & & 46.782 & 6235.0 & 14.200 & 13.452 & 0.000 & \\
\hline 100 & 0.777 & 643 & & 48.023 & 1069.4 & 13.746 & 12.916 & 0.001 & \\
\hline 110 & 3.416 & 578 & & 49.294 & 267.15 & 13.296 & 12.386 & 0.002 & \\
\hline 120 & 11.316 & 520 & & 50.656 & 87.646 & 12.878 & 11.895 & 0.008 & \\
\hline 130 & 30.379 & 467 & & 52.141 & 35.106 & 12.468 & 11.423 & 0.020 & \\
\hline 140 & 69.520 & 420 & & 53.770 & 16.312 & 12.043 & 10.958 & 0.045 & \\
\hline 145.16 & 101.360 & 398 & & 54.676 & 11.495 & 11.810 & 10.715 & 0.065 & normal boiling point \\
\hline 150 & 140.622 & 378 & & 55.571 & 8.473 & 11.580 & 10.485 & 0.089 & \\
\hline 160 & 258.150 & 339 & -1768 & 57.596 & 4.789 & 11.061 & 10.003 & 0.162 & \\
\hline 170 & 438.670 & 305 & +4395 & 59.937 & 2.883 & 10.465 & 9.498 & 0.268 & \\
\hline 180 & 700.435 & 275 & 7608 & 62.748 & 1.817 & 9.780 & 8.977 & 0.416 & \\
\hline 190 & 1063.240 & 248 & 9139 & 66.257 & 1.183 & 8.991 & 8.438 & 0.611 & \\
\hline 200 & 1548.702 & 224 & 9705 & 70.18 & 0.783 & 8.073 & 7.869 & 0.860 & \\
\hline 210 & 2181.271 & 202 & 9733 & 76.10 & 0.522 & 7.015 & 7.301 & 1.167 & \\
\hline 220 & 2990.970 & 183 & 9472 & 85.05 & 0.341 & 5.711 & 6.653 & 1.540 & \\
\hline $227.5^{b}$ & $3742^{b}$ & 170 & 9175 & $(140)^{c}$ & $(0.140)^{c}$ & (0) & & & critical point \\
\hline
\end{tabular}

${ }^{a}$ With the exception of the virial coefficients $B$ and $C$, which are independent of pressure, all of the properties listed refer to saturation conditions. $b$ From ref 13 . ${ }^{c}$ From ref 14.

paring the trend shown by this plot with that for other substances (11). In this manner a figure of $d=1.0$ was obtained for $\mathrm{CF}_{4 \text { - }}$ The Chueh and Prausnitz expression was then used to estimate $C$ values down to $160 \mathrm{~K}$, at which temperature the calculated values start to become negative, and therefore the method is no longer reliable. The neglect of the third virial coefficient below $160 \mathrm{~K}$ should not give rise to any serious error in the derived quantities since the pressures involved never exceed $0.2 \mathrm{MPa}$.

From this information the internal configurational energy of the saturated vapor $U_{g}^{*}$ can also be derived from the expression

$$
U_{g}^{*}\left(T, P_{s}\right)=-\left|B_{1}\left(P_{s}-B P_{s}^{2} / R T\right)+C_{1}\left(2 P_{2}^{2} / R T\right)\right|
$$

For pressures above $2 \mathrm{MPa}$, the inclusion of terms involving the fourth virial coefficient $D$ in eq 4 and 9 did in no case change the calculated values of $U_{1}^{*}$ and $U^{*}$ by more than $\sim 1 \%$. $D$ was obtained from a linear extrapolation of the experimental values given by Douslin et al. (9).

The molar volumes of the liquid $V_{1}$ were derived from the experimental work of Terry et al. (5). A small correction was applied to their values, since these are now recognized to embody a systematic error and as a result to be $0.4 \%$ too high (10). Outside the temperature range to which their expression applies, estimates of $V$, were made by using the law of the rectilinear diameter with parameters given in their paper and values of $V_{g}$ calculated as explained above.

Since experimental results are also avallable for the velocity of sound, $u$, in liquid carbon tetrafluoride (12), we have calculated the mechanical coefficients for this substance. The procedure adopted was much the same as that indicated by Blagoi et al. (12). First, the velocity of sound was used to estimate the adiabatic compressibility $\kappa_{S}$

$$
\kappa_{s}=V_{1} /\left(M u^{2}\right)
$$

where $M$ is the mass of unit amount of substance, and also the quantity $\gamma$

$$
\gamma=1+T \alpha_{p}^{2} u^{2} / C_{p} \simeq 1+T \alpha_{\sigma}^{2} u^{2} / C_{\sigma}
$$

where the subscript $\sigma$ refers to saturation conditions. Values of the expansion coefficient $\alpha_{\sigma}$

$$
\alpha_{\sigma}=\left(1 / N_{1}\right)\left(\partial V_{1} / \partial T\right)_{\sigma}
$$

were derived from the (corrected) expression of Terry et al., while those of the saturation heat capacity $C_{\sigma}$ were taken from the accurate calorimetric work of Smith and Pace (7). From these values of $\kappa_{S}$ and $\gamma$, the isothermal compressibility $\kappa_{T}$

$$
\kappa_{T}=-\left(1 / V_{1}\right)\left(\partial V_{1} / \partial P\right)_{T}
$$

could be obtained, since

$$
\kappa_{T}=\gamma \kappa_{S}
$$

$\kappa_{T}$ was in turn used to estimate the isobaric expansion coefficient $\alpha_{p}$ through the relationship

$$
\alpha_{P}=\alpha_{\sigma}+\kappa_{T} \gamma_{\sigma}
$$

where $\gamma_{\sigma}$ is the temperature coefficient of the vapor pressure

$$
\gamma_{\sigma}=(\partial P / \partial T)_{\sigma}
$$

taken from our own work (4). Next, the heat capacity of the liquid at constant pressure $C_{p}$ was estimated from the expression

$$
C_{P}=C_{\sigma}+T V_{1} \alpha_{P} \gamma_{\sigma}
$$

In the temperature range over which reliable values of $C_{\sigma}$ for $\mathrm{CF}_{4}$ are available $(90$ to $\sim 145 \mathrm{~K}$ ), the pressures involved are always low, and therefore $C_{p}$ does not differ significantly from $C_{\sigma}$, nor $\alpha_{p}$ from $\alpha_{\sigma}$. No iteration is then necessary to refine the first set of values obtained from the calculations outlined above. Finally, the heat capacity of the liquid at constant volume $C_{V}$ was estimated from

$$
C_{P}=\gamma C_{V}
$$

and the thermal pressure coefficient $\gamma_{v}$ was obtained from

$$
\gamma_{V}=\alpha_{P} / \kappa_{T}
$$

\section{Results and Discussion}

The results of the calculations outlined above are shown in Tables I and II.

It would clearly be helpful if an assessment could be made of the reliability of at least some of the values in these tables. As far as the second virial coefficients are concerned, such an assessment should obviously be made at lower temperatures for which the correctness of the extrapolations that we have made (from experimental $B$ values in the range from $\sim 200$ to $\sim 320 \mathrm{~K}$ ) could perhaps be questioned. The method proposed by Curtiss and Hirschfelder (15) to estimate $B$ from a combination of the Clausius-Clapeyron and the virial equation of state (truncated after the second term) seems to be a reasonable choice for comparison. By using this, one arrives at a value 
Table II. Mechanical, Adiabatic, and Thermal Coefficients of Saturated Liquid Carbon Tetrafluoride

\begin{tabular}{|c|c|c|c|c|c|c|c|c|c|}
\hline$T, \mathrm{~K}$ & $\begin{array}{c}10^{3} \alpha_{\sigma} \\
\mathrm{K}^{-1}\end{array}$ & $10^{3} \alpha_{\mathrm{P}}$, & $\begin{array}{l}10^{3} \mathrm{KS}_{\mathrm{S}}, \\
\mathrm{MPa}^{-1}\end{array}$ & $\begin{array}{l}10^{3} \kappa_{T} \\
\mathrm{MPa}^{-1}\end{array}$ & $\begin{array}{c}\gamma_{\sigma}, \\
\mathrm{kPa} \mathrm{K}^{-1}\end{array}$ & $\begin{array}{c}\gamma \mathrm{V} \\
\mathrm{MPa} \mathrm{K}^{-1}\end{array}$ & $\gamma$ & $\begin{array}{c}C_{P}, \mathrm{~J} \\
\mathrm{~mol}^{-1} \mathrm{~K}^{-1}\end{array}$ & $\begin{array}{c}C_{V}, \mathrm{~J} \\
\mathrm{~mol}^{-1} \mathrm{~K}^{-1}\end{array}$ \\
\hline $\begin{array}{l}89.56 \\
90 \\
100 \\
110 \\
120 \\
130 \\
140 \\
145.16 \\
150 \\
160 \\
170 \\
180 \\
190 \\
200 \\
210 \\
220 \\
227.5\end{array}$ & $\begin{array}{l}2.70 \\
2.69 \\
2.59 \\
2.65 \\
2.80 \\
2.98 \\
3.18 \\
3.30 \\
3.42 \\
3.76 \\
4.25 \\
4.97 \\
5.98\end{array}$ & $\begin{array}{l}2.69 \\
2.59 \\
2.65 \\
2.80 \\
2.98 \\
3.19 \\
3.32\end{array}$ & $\begin{array}{r}0.44 \\
0.44 \\
0.51 \\
0.60 \\
0.71 \\
0.85 \\
1.05 \\
1.17 \\
1.32 \\
1.67 \\
2.17 \\
2.93 \\
4.21 \\
6.45 \\
11.61 \\
27.40\end{array}$ & $\begin{array}{l}0.83 \\
0.93 \\
1.09 \\
1.33 \\
1.62 \\
2.01 \\
2.25\end{array}$ & $\begin{array}{r}0.0233 \\
0.0253 \\
0.1285 \\
0.4525 \\
1.225 \\
2.736 \\
5.291 \\
7.112 \\
9.171 \\
14.611 \\
21.797 \\
30.885 \\
42.034 \\
55.462 \\
71.541 \\
91.106 \\
111.275\end{array}$ & $\begin{array}{l}3.24 \\
2.78 \\
2.43 \\
2.11 \\
1.84 \\
1.59 \\
1.48\end{array}$ & $\begin{array}{l}1.882 \\
1.819 \\
1.824 \\
1.871 \\
1.906 \\
1.913 \\
1.921\end{array}$ & $\begin{array}{l}78.0 \\
77.1 \\
77.2 \\
77.4 \\
78.2 \\
79.5 \\
80.3\end{array}$ & $\begin{array}{l}41.5 \\
42.4 \\
42.3 \\
41.4 \\
41.0 \\
41.5 \\
41.8\end{array}$ \\
\hline
\end{tabular}

for $B$ at $89.56 \mathrm{~K}$-the triple-point temperature $T_{\text {tr }}$ of $\mathrm{CF}_{4}$ which is only $3 \%$ in excess of that obtained from eq 8 . Moreover, at the normal boiling-point temperature $\left(T_{b}=145.16\right.$ $K)$ the deviation between the values of $B$ arrived at by the two methods is even less (4): $0.5 \%$. It is well-known that the method of Curtiss and Hirschfelder relles on the accuracy of the vapor-pressure equation since the $B$ coefficients derived in this manner strongly depend on $\mathrm{d} P / \mathrm{d} T$. The agreement obtained in the present case can thus perhaps be simultaneously regarded as supporting our vapor-pressure equation, and hence glving confidence in the calculated $\gamma_{\sigma}$ values.

The expression of Chueh and Prausnitz with $d=1.0$ reproduces the experimental values of $C$ at both -70 and $0^{\circ} \mathrm{C}$ within the experimental uncertainty quoted by Lange and Stein (8). The values of $V_{g}$, the molar volume of the gaseous phase, listed in Table I should therefore be reasonably accurate.

We measured the molar volume of liquid carbon tetrafluoride (10) at the triple-point temperatures of hydrogen chloride ( $T_{\mathrm{tr}}$ $=158.95 \mathrm{~K})$ and hexafluoroethane $\left(T_{\mathrm{t}}=173.11 \mathrm{~K}\right)$ in a pyknometer which had been calibrated at 158.95 and $182.32 \mathrm{~K}$ (the triple-point temperature of nitrous oxide) by using a highly purified sample of ethane and the density results of Haynes and Hza (16). At both temperatures our results were $0.4 \%$ lower than those obtalned from the work of Terry et al. (5). A discrepancy of almost the same magnitude has been found for several substances (17). Accordingly, allowance was made for this, so that the values of $V_{1}$ reported in Table I (and hence those of $\alpha_{\sigma}$ ) should be of adequate quallty for our main purposes.

To judge the quality of the $\Delta H_{v}$ values llsted in Table I is perhaps difficult. Although carbon tetrafluoride is an important substance in industry, and otherwise, as far as we are aware only one measurement of sufficient accuracy appears to have been reported for its enthalpy of vaporization. This is that by Smith and Pace (7), whose reported value at the normal bolling point is $\Delta H_{v, b}=11814 \pm 5 \mathrm{~J} \mathrm{~mol}^{-1}$. Use of the ClauslusClapeyron equation and our vapor-pressure equation glves $11810 \mathrm{~J} \mathrm{~mol}^{-1}$, which is virtually the same figure, as already mentioned. We have also tried to assess the quality of the estimated $\Delta H_{v}$ at the triple-point temperature of $\mathrm{CF}_{4}(89.56 \mathrm{~K})$ by using it in an evaluation from vapor-pressure data of the enthalpy of fusion $\Delta H_{f}$. Several authors $(7,18,19)$ have reported experimental values of $\sim 0.70 \mathrm{~kJ} \mathrm{~mol}^{-1}$ for this quantlty. Simon et al. (20) estimated the enthalpy of sublimation of carbon tetrafluoride at its own triple-polnt temperature as $\Delta H_{a b}=14.73 \mathrm{~kJ} \mathrm{~mol}^{-1}$, from their measured vapor pressure of the solld. The difference between this value and ours $\left(\Delta H_{v}(89.56 \mathrm{~K})=14.26 \mathrm{~kJ} \mathrm{~mol}^{-1}\right)$ gives $\Delta H_{\mathrm{f}}=0.47 \mathrm{~kJ} \mathrm{~mol}^{-1}$, whlch can be regarded as being in reasonable agreement with the experimental value, especially if one takes into account that the $\Delta H_{f}$ so calculated is only $\sim 3 \%$ of the terms from whose difference it was obtalned. One further test on our reported $\Delta H_{v}\left(T_{t}\right)$ is by using the thermodynamic relationship

$$
\left(\partial \Delta H_{v} / \partial T\right)_{P} \approx C_{P, \theta}-C_{P, I}
$$

From the experimental $\Delta H_{v, b}$ and the $C_{P, 1}$ from ref 7 , and by assuming the perfect-gas heat capacity for gaseous $\mathrm{CF}_{4}$ in the range from $T_{t}$ to $T_{b}$, one obtains $\Delta H_{v}\left(T_{t}\right)=14.76 \mathrm{~kJ} \mathrm{~mol}^{-1}$. This is only $\sim 3 \%$ higher than the figure reported in Table I.

Since the quantities involved in the calculation of $U^{*}$ and $U^{*}$. all seem reasonably trustworthy, one should expect the same for the calculated values of both configurational internal energles.

In view of the particular behavior of substances in the critical region, no attempt was made to extend the calculations to the critical temperature. However, it is interesting to note that the values calculated from $\theta q 4$ and $\theta$ for $U_{1}^{*}$ and $U_{g}^{*}$ at $T_{c}=$ $227.5 \mathrm{~K}$ are -1.75 and $-1.87 \mathrm{~kJ} \mathrm{~mol}^{-1}$, respectively, in good agreement with each other.

Little can be said about the remaining quantties listed in Table II. The agreement with values calculated by Blagoi et al. (12) is variable. While the differences remain within their quoted uncertainties for $C_{V}$ and $\gamma$, the agreement is poor for $\kappa_{T}$ and $\kappa_{S}$. Since the same values of the velocity of sound, $u$, were used in both the Russian work and in ours, the difference between the two sets of $\kappa_{s}$ values can only be assigned to the possible use of rather poor estimates of $V_{1}$ by Blagol and coworkers, especially at the lower temperatures for whlch comparison with our figures could be made. (The difference between the two sets of $\kappa_{s}$ values at 140,120 , and $100 \mathrm{~K}$ is respectlvely 0,20 , and $40 \%$ of their reported $\kappa_{s}$ flgures).

Finally, our listed values for the expansion coefficients may be compared with $\alpha=(2.59 \pm 0.04) \times 10^{-3} \mathrm{~K}^{-1}$ for $T$ from 94 to $107 \mathrm{~K}$, as extracted from the work of Croll and Scott (21).

\section{Llterature Clted}

(1) Powles, J. G.; Evans, W. A. B.; McCrath, E.; Gubblns, K. E.; Murad, S. Mol. Phys. 1079, 38, 883.

(2) Nunes da Ponte, M.; Staveley, L. A. K. J. Chem. Thermootyn., in press.

(3) Gubbins, K. E.; Lobo, L. Q.; Staveley, L. A. K., unpubilshed data, 1979-1980.

(4) Lobo, L. Q.; Staveley, L. A. K. Gryogenies 1070, 19, 336.

(5) Terry, M. J.; Lynch, J. T.; Bunclark, M.; Mansell, K. R.; Staveley, L. A. K. J. Chem. Thermodyn. 1980, 1, 413.

(6) Ambrose, D. Proc. NPL Conf:: Chem. Thermodyn. Data Fluids Fluid Mxtures: Their Estlm., Correl. Use, $19781070,193$.

(7) Smlth, J. H.; Pace, E. L. J. Phys. Chom. 1989, 73, 4232.

(8) Lange, H. B., Jt.; Stein, F. P. J. Chem. Eng. Data 1970, 15, 56.

(9) Doualln, D. R.; Harrison, R. H.; Moore, R. T.; McCullough, J. P. J. Chem. Phys. 1081, 35, 1357.

(10) Lobo, L. Q. Ph.D. Thesis, Untversity of Oxford, Oxford, England, 1979 
(11) Chuoh, P. L.; Prausnitz, J. M. AIChE J. 1967, 13, 898

(12) Blagoi, Y. P.; Butko, A. E.; Mikhallenko, S. A.; Yakuba, V. V. Russ. J Phys. Chem. (Engl. Transl.) 1968, 42, 564.

(13) Engineering Sclences Data Unit, Index 1977-78, ESDU: London, 1977 , p 248.

(14) Kudchadker, A. P.; Alani, G. H.; Zwolinski, B. J. Chem. Rev. 1968, 68,659 .

(15) Curtiss, C. F.; Hirschfelder, J. O. J. Chem. Phys. 1942, 8, 491

(16) Haynes, W. M.; Hiza, M. J. J. Chem. Thermodyn. 1977, 9, 179

(17) Nunes da Ponte, M.; Streett, W. B.; Staveley, L. A. K. J. Chem. Ther modyn. 1978, 10,151.
(18) Eucken, A.; Schröder, E. Z. Phys, Chem., Abt. B 1938, 41, 307.

(19) Enokido, H.; Shlnoda, T.; Mashlko, Y. Bull.' Chem. Soc. Jpn. 1989, 42,3415 .

(20) Simon, M.; Knobler, C. M.; Duncan, A. G. Cryogenics 1967, 7, 138

(21) Croll, I. M.; Scott, R. L. J. Phys. Chem. 1958, 62, 954.

Received for review February 4, 1981. Accepted Aprll 27, 1981. L.Q.L. is grateful to The Royal Society (London) and Academia das Ciências de Lisboa for the award of a Visiting Fellowship in the European Science Exchange Programme.

\title{
Measurement of the Electrical Conductivites of Molten $\mathrm{NaNO}_{3}-\mathrm{KNO}_{3}-\mathrm{NaNO}_{2}$ and Molten LiF-NaF-KF by Displacing the Positions of Electrodes
}

\author{
Yohji Uchlyama" ${ }^{\dagger}$ and Kazutaka Kawamura \\ Research Laboratory for Nuclear Reactors, Tokyo Institute of Technology 2-12-1, O-okayama, Meguro-ku, Tokyo 152, Japan
}

In order to obtain the true electrical conductance of solution in the ac bridge method, it is necessary to ellminate both the trequency effect resulting from clrcultry and that of the metal/salt interfacial impedance. In this study we devised the apparatus of electrical conductances so that the interfaclal impedance could be neglected by subtracting the measured resistances between two positions of electrodes which were moved through a caplilary cell by using a micrometer. With this method it has been found that the same solution resistance could be obtained at any frequency without extrapolating infinitely the measured resistance against the reciprocal of frequency. The experiments were carrled out for molten $\mathrm{NaNO}_{3}, \mathrm{KNO}_{3}$, heat transfer salt (HTS) (a mlxture of molten $\mathrm{NaNO}_{3}-\mathrm{KNO}_{3}-\mathrm{NaNO}_{2}, 7: 44: 49$ mol \%), and FLINAK (a mlxture of molten LIF-NaF-KF ternary eutectic, 46.5:11.5:42.0 mol \%).

\section{Introduction}

For the measurement of electrical conductivity of molten salts, two different types of conductance cells have been used: the capillary cell and the immersion cell. In general, molten salts except covalent compound like the mercuric halides have higher specific conductivities than those of aqueous electrolytes. When one takes into account that the resistance value of the immersion cell $(1 \sim 10 \Omega)$ is low as compared with that of the capillary cell $(500 \sim 1500 \Omega)$, polarization effects at the surface of the electrodes of the immersion cell will make accurate measurement of resistance values practically impossible. For accurate measurement of resistance, the ac conductance bridge techniques with high cell constants are readily applicable as compared with the immersion cell.

The measured resistance in the ac bridge technique also contains the frequency effects resulting from circuitry and the electrode impedance resulting from polarization effects at the electrodes, which depend on the frequency of the applied alternating voltage. If the former effects are neglected, one can consider that the measured resistance, $R_{m}$, and the resistance associated with the electrode/solution interface, $R_{2}$, depend on the frequency of the applied current.

$$
R_{\mathrm{m}}=R_{\mathrm{s}}+R_{\mathrm{z}}
$$

TPresent address: Central Research Instltute of Electrical Power Industry, Otemachi Building, 1-6-1 Ohtemachi, Chiyoda-hu, Tokyo 100, Japan.

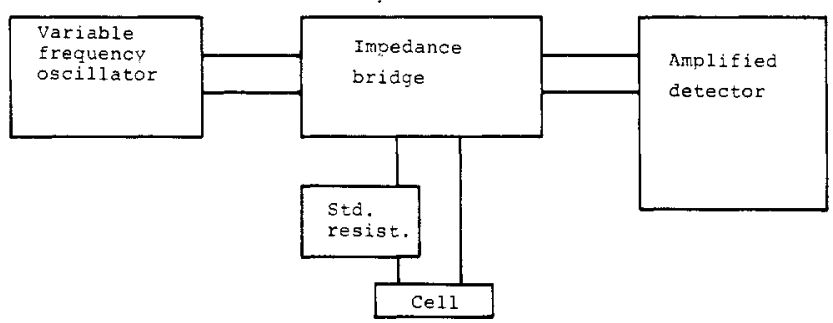

Figure 1. Experimental assembly for electrical conductivity.

$R_{2}$ must be either corrected or eliminated experimentally so that $R_{\mathrm{m}}$ represents the true resistance of the solution.

In the measurements of molten salts, a value of $R_{\mathrm{m}}$ is generally extrapolated to infinite frequency with $f^{-1 / 2}$ or $f^{-1}$, where $R_{z}$ is assumed to be zero. However, it has been reported that, in molten nitrates (1) and chlorides and fluorides (2), $R_{\mathrm{m}}$ is nonlinear in $f^{-1 / 2}$ or $f^{-1}$, with resistance approaching a nearly constant value at high frequencies. Although the effects of the metal/salt interfacial impedance have been studied by some investigators $(3,4)$, the phenomena at the interface are too complicated to clarify. This polarization effect appears to lead to the error of specific conductivity of molten salts, especially fluoride melts which exhibit high ionic conductivity. The purpose of the present work is to eliminate the polarization impedance.

We devised an apparatus of electrical conductivities so that two electrodes could be moved through a capillary cell by using a micrometer. The difference of measured resistances between two arbitrarily chose electrode positions shows the true solution resistance of a melt, $R_{\mathrm{s}}$, since the polarization impedance could be considered to have the same value at any position and be neglected by subtracting.

In order to check this method, the specific conductivities of a 1.0 demal $\mathrm{KCl}$ solution and of molten $\mathrm{NaNO}_{3}$ and $\mathrm{KNO}_{3}$ were measured. Then, those of heat transfer salt and FLINAK were measured since they are stable over a relatively wide range of temperatures and, to our knowledge, there are no available data for FLINAK. With this method we found that the same solution resistance, $R_{s}$, could be obtained at any frequency and that it was not necessary to extrapolate the measured resistance with respect to frequency.

\section{Experimental Section}

Figure 1 shows a schematic outline of the bridge circuit ap- 